\title{
Different Vocal Parameters Predict Perceptions of Dominance and Attractiveness
}

\author{
Carolyn R. Hodges-Simeon • Steven J. C. Gaulin • \\ David A. Puts
}

Published online: 27 November 2010

C The Author(s) 2010. This article is published with open access at Springerlink.com

\begin{abstract}
Low mean fundamental frequency $\left(F_{0}\right)$ in men's voices has been found to positively influence perceptions of dominance by men and attractiveness by women using standardized speech. Using natural speech obtained during an ecologically valid social interaction, we examined relationships between multiple vocal parameters and dominance and attractiveness judgments. Male voices from an unscripted dating game were judged by men for physical and social dominance and by women in fertile and non-fertile menstrual cycle phases for desirability in shortterm and long-term relationships. Five vocal parameters were analyzed: mean $F_{0}$ (an acoustic correlate of vocal fold size), $F_{0}$ variation, intensity (loudness), utterance duration, and formant dispersion ( $D_{f}$, an acoustic correlate of vocal tract length). Parallel but separate ratings of speech transcripts served as controls for content. Multiple regression analyses were used to examine the independent contributions of each of the predictors. Physical dominance was predicted by low $F_{0}$ variation and physically dominant word content. Social dominance was predicted only by socially dominant word content. Ratings of attractiveness by women were predicted by low mean $F_{0}$, low $D_{f}$, high intensity, and attractive word content across cycle phase and mating context. Low $D_{f}$ was perceived as attractive by fertile-phase women only. We hypothesize that competitors and potential mates may attend more strongly to different components of men's voices because of the different types of information these vocal parameters provide.
\end{abstract}

Keywords Dominance $\cdot$ Mate choice $\cdot$ Sexual selection $\cdot$ Voice $\cdot$ Voice pitch $\cdot$ Formant dispersion

C. R. Hodges-Simeon · S. J. C. Gaulin

Department of Anthropology, University of California, Santa Barbara, CA 93106, USA

D. A. Puts $(\bowtie)$

Department of Anthropology, Pennsylvania State University, University Park, PA 16802, USA

e-mail: dap27@psu.edu 
Speech is complex, both semantically and acoustically. A number of distinct vocal parameters have been shown to affect interpersonal perceptions in humans (e.g., Banse and Scherer 1996; Feinberg et al. 2005; Puts et al. 2007; Scherer 1986; Zuckerman and Miyake 1993). Evolutionists have focused primarily on the effect of low-sounding vocalizations on the dynamics of male-male competition and female mate choice (via judgments of dominance and attractiveness); however, human speech varies along many dimensions. The purpose of the present paper is to examine relationships between these evolutionarily relevant judgments and multiple vocal parameters by using naturally varying, unscripted speech generated in an ecologically valid social interaction. Many studies of this kind use vocal stimuli that are unnaturally invariant in content and motivation, with all speakers uttering precisely the same, often socially irrelevant, phrase (e.g., Bruckert et al. 2006; Feinberg et al. 2006). Although this methodology permits experimental control, it filters out the actual contexts in which courtship and competition occur. The generalizability and external validity of such tightly controlled results depend on whether the effects they reveal persist in natural speech. Control for content is of course required, but our approach is to control semantic content statistically rather than experimentally.

In addition, the existing literature has focused primarily on the ways in which males and females converge in their response to acoustic parameters; however, there are adaptive reasons why males and females may not target the same types of acoustic information. In terms of the force of sexual selection, one might expect that men and women would have evolved to be responsive to different male attributes. Both sexes might form alliances with males, but beyond this, males are potential sexual competitors for men whereas they are potential mates for women. To the extent that competitive traits might be revealed by different aspects of voice than would traits revealing of mate quality, these different priorities may favor men and women attending to different attributes of men's voices.

The goal of this paper is to examine the independent and joint effects of four acoustic parameters - formant dispersion, mean fundamental frequency, fundamental frequency variation, and intensity - plus duration and semantic content, in natural speech. Separate, parallel analyses are performed to assess the effects of each of these six variables on male perceptions of dominance and female perceptions of attractiveness.

\section{Formant Dispersion $\left(D_{f}\right)$}

Low-sounding vocalizations are the product of multiple acoustic parameters, including compact formant frequencies (low "formant dispersion" $\left[D_{f}\right]$; Fitch 1997) and low fundamental frequency $\left(F_{0}\right) . D_{f}$ is a measure of the average spacing between the formants, which are energy peaks in the harmonic spectrum (Feinberg 2008; Fitch and Giedd 1999). Lower formants and formant dispersion give a more "resonant" quality to the voice. $D_{f}$ is a function of the length and shape of the vocal tract (or the depth of the larynx in the throat), which is the space through which sound waves must travel from the vocal folds through the oral cavity. Until puberty, vocal tract length scales with body size growth without any sexual dimorphism 
(Vorperian et al. 2005). At puberty, modulated by an increase in testosterone, males' larynges begin to descend farther than females' (Fitch and Giedd 1999). The eventual product of this laryngeal alteration is a longer vocal tract and the resultant "deeper," more resonating voice among adult males. On average, the vocal tract is about 15\% longer in adult males (Fant 1960) and is correlated with height (Fitch 1997). This results in sex differences in $D_{f}$, with males exhibiting formants of lower frequency and dispersion (Collins 2000; Feinberg et al. 2005; Gonzalez 2004), and constitutes the basis for the indexical value of low $D_{f}$.

Recent findings show that experimentally lowering $D_{f}$ elevates dominance ratings (Puts et al. 2007; Wolff and Puts 2010), and low $D_{f}$ is associated with assessments of larger size, muscularity, masculinity, and age (Collins 2000; Feinberg et al. 2005). However, studies investigating the relationship between men's $D_{f}$ and women's judgments of male attractiveness have produced mixed or inconclusive results (Collins 2000; Feinberg et al. 2005).

\section{Fundamental Frequency $\left(F_{0}\right)$}

Although $F_{0}$ was first hypothesized to indicate large body size (e.g., Morton 1977), the evidence for relationships between body size parameters and average $F_{0}$ in humans is inconsistent (Bruckert et al. 2006; Collins 2000; Gonzalez 2004; Evans et al. 2006; Lass and Brown 1978; van Dommellen 1993; van Dommellen and Moxness 1995). On the other hand, $F_{0}$ 's dependence on testosterone (T) may constrain it as an honest signal. Working through androgen receptors in the vocal folds (Newman et al. 2000), testosterone lengthens and thickens the vocal folds, lowering mean $F_{0}$ in pubertal males (Harries et al. 1997; Hollien et al. 1994; Titze 1989) and in adults undergoing $\mathrm{T}$ treatment (Need et al. 1993). Given that $\mathrm{T}$ has been linked to aggressive responses to challenges (Archer 2006), cues of $\mathrm{T}$ dosage such as low $F_{0}$ (Dabbs and Mallinger 1999; Evans et al. 2008) may usefully predict a competitor's aggressive potential.

Because only well-buffered individuals can tolerate the immunosuppressant effects of T (Folstad and Karter 1992; Grossman 1985), mean $F_{0}$ may also indicate parasite resistance (Feinberg et al. 2005; Puts 2005). Coevolutionary contests with parasites can maintain heritability in parasite resistance and its indicators, and thus the basis for good-genes mate choice in hosts (Hamilton and Zuk 1982). To the degree that immunocompetence and $F_{0}$ remained heritable over human evolution, women's judgments of male vocal attractiveness may have evolved to recruit these qualities for their offspring. Indeed, mean $F_{0}$ is heritable (Debruyne et al. 2002), and women's preferences for male voices within a certain $F_{0}$ range appear suited for the function of sire choice. Men with deeper voices are perceived as more attractive than those with higher voices (Feinberg et al. 2005, 2006; Jones et al. 2010; Puts 2005), and men with attractive voices have greater mating success than those with lessattractive voices (Hughes et al. 2004). Further, women are more attracted to low- $F_{0}$ male voices when near ovulation (Puts 2005; Feinberg et al. 2006) and in short-term mating contexts (Puts 2005). These preferences may increase the reproductive success of men with low voice pitch; in a sample of Western undergraduates, men with lower voice pitch reported more sexual partners in the past year than those with 
higher voice pitch (Puts 2005), and in a study of hunter-gatherers, men with lower voice pitch reported more offspring (Apicella et al. 2007).

People also associate low $F_{0}$ with physical and social characteristics that contribute to success in competitive encounters (i.e., relative dominance); males with lower $F_{0}$ are rated as older, taller, and heavier (Collins 2000; Feinberg et al. 2005; Lass and Brown 1978). In addition, experimentally lowering $F_{0}$ increases perceptions of dominance (Jones et al. 2010; Puts et al. 2007; Wolff and Puts 2010).

\section{Variation in $\boldsymbol{F}_{0}$}

Many of the above-cited studies measure $F_{0}$ with software that repeatedly samples a digital speech segment and averages across the samples. While this method effectively captures mean $F_{0}$, it may obscure state-dependent patterns of variation. Vocal fold tension is under significant volitional control, often in service of linguistic expression (Ohala 1983; Johnstone and Scherer 2005); however, it is also subject to nonvolitional influence (Scherer 1986; Johnstone and Scherer 2005) via changes in respiratory patterns, diaphragmatic and abdominal tension, and hormonal status, all of which are influenced by affective state (Titze 1994). It is therefore important not only to understand vocal indicators of fixed or "inherent" quality, but also those that index fluctuating states between and within social contexts.

Because vocal signals reflect state-dependent changes (Scherer 1986), they may be particularly relevant in domains of social life in which relationships shift situationally, such as dominance interactions. Changes in mean $F_{0}$ have been shown to vary across contexts depending on the characteristics of the signaler; men who perceive themselves to be dominant tend to lower their mean $F_{0}$ when addressing a competitor, whereas men who perceive themselves to be subordinate tend to raise their mean $F_{0}$ (Puts et al. 2006).

The extent to which $F_{0}$ fluctuates over an utterance may also index socially relevant state-dependent changes (Scherer 1986). $F_{0}$ variation quantifies one aspect of prosodic individual differences; it captures the extent of "highs" and "lows" in speech (this is in contrast to jitter, which measures shorter cycle-to-cycle variation in $F_{0}$; Baken 1987). Perceptually, low $F_{0}$ variation has a monotone quality, whereas high $F_{0}$ variation can have a sing-song character. Adults are more likely to exaggerate $F_{0}$ variation when speaking to infants than when speaking to other adults (Trainor et al. 2000), suggesting that high variation in $F_{0}$ may be associated with friendly and appeasing contexts. However, high $F_{0}$ variation is also characteristic of high-activation emotions (e.g., panic fear and "hot" anger) (Banse and Scherer 1996). Among cotton-top tamarins (Saguinus oedipus oedipus), high $F_{0}$ variation is indicative of a proclivity to engage in playful wrestling (Goedeking 1988). If high $F_{0}$ variation is used to affiliate, then lack of $F_{0}$ modulation (i.e., monotonic vocalization) may be used to intimidate.

Riding et al. (2006) demonstrated a trend toward increasing attractiveness in men's voices with decreasing $F_{0}$ variation, although the effect was not significant. In contrast, other studies have shown that moderate to high $F_{0}$ variation is associated with higher social attractiveness ratings (Ray et al. 1991; Zuckerman and Miyake 1993). Some of this discrepancy may be accounted for by examining attractiveness 
ratings across menstrual phases and imagined mating contexts, both of which affect women's preferences for putative indicators of genetic quality (Penton-Voak et al. 1999; Puts 2005). Mean $F_{0}$ has been argued to reflect genetic quality, and its attractiveness has been shown to vary with female fertility and mating context in a way that supports such a perspective (Puts 2005; Feinberg et al. 2006). If $F_{0}$ variation also reflects genetic quality, then preferences for it might also change with cycle phase and mating context, but if $F_{0}$ variation does not track heritable fitness differences in men, then there should be no difference in preferences for it across cycle phase and mating context.

\section{Intensity}

Intensity is a measure of power per unit of area and roughly corresponds to the psychoacoustic term "loudness" (Baken 1987). Individuals can vary intensity by changing the amount of lung pressure and the size of the glottal (vocal fold) opening (Titze 1994). Changes in intensity are used to communicate semantic (i.e., to emphasize certain syllables and words) and emotional information (Banse and Scherer 1996). Like high $F_{0}$ variation, intensity is a characteristic of high activation emotions - fear, anger, and joy (Banse and Scherer 1996). More confident individuals speak with greater intensity (Kimble and Seidel 1991), and high intensity is associated with perceptions of dominance (Aronovitch 1976; Scherer et al. 1973).

\section{The Present Study}

A central goal of the present research is to assess the extent to which these existing findings on the relationships between acoustic parameters, on the one hand, and dominance and attractiveness judgments, on the other, generalize to ecologically valid competitive and courtship contexts. It is possible that subtle acoustic variation is salient only against the background of fixed content. In addition, our research format allows a comparison of the relative influence of acoustic and semantic variables on dominance and attractiveness judgments. Finally, for theoretical reasons outlined above, male judgments of dominance and female judgments of attractiveness might be expected to vary in response to different acoustic parameters. Females might attend to invariant characteristics signaling genetic quality, whereas males might attend to situational factors that predict the outcome of competitive encounters. All of these possibilities are evaluated first in univariate and then in multivariate contexts.

\section{Method}

Acoustic stimuli and audio ratings were collected by Puts and colleagues (Puts 2005; Puts et al. 2006). Content ratings of transcriptions of the same audio recordings were collected for the present study and provided a control for semantic variation among speakers. 


\section{Subjects}

Participating in this study were 264 self-reported heterosexual males and 177 selfreported heterosexual females, distributed as follows: 111 male "participants" (ages 18$24, M=18.9$ years, $\mathrm{SD}=1.2$ ), 86 male "audio raters" (ages $18-28, M=20, \mathrm{SD}=2.1$ ), 142 female "audio raters" (ages 18-30, $M=19.1, \mathrm{SD}=1.7), 67$ male "content raters" (ages 18-26, $M=19.2, \mathrm{SD}=1.6$ ), and 35 female "content raters" (ages 18-37, $M=19.5$, $\mathrm{SD}=2.7)$. All participants spoke native, minimally accented American English. Additionally, both participants and audio raters were sampled from the same community (University of Pittsburgh undergraduates). Content raters were similarly aged male and female undergraduates at the University of California, Santa Barbara.

\section{Procedure}

\section{Acoustic Stimuli}

In the first part of the study, male participants competed in a mock dating-game scenario modeled after Simpson et al. (1999; see Puts 2005 and Puts et al. 2006 for methodological details). Before learning the dating-game format, each participant was asked to read a control passage out loud (the Rainbow Passage). Participants were next instructed to describe themselves to their potential date ("courtship recording"). They were then asked to address their male competitor and describe why they might be respected or admired by other males ("competitive recording"). All participants' responses were recorded using GoldWave digital audio software. After the courtship and competitive phases, participants were asked to rate their own and their competitor's physical and social dominance on a Likert scale. In accordance with procedures described by Mazur et al. (1994), raters were told that "a [socially] dominant person tells other people what to do, is respected, influential, and often a leader; whereas submissive people are not influential or assertive and are usually directed by others." Raters were then asked to assess social dominance by marking a visual analogue scale ranging from "extremely dominant" to "extremely submissive." Regarding physical dominance, raters were asked to mark a visual analogue scale anywhere from "strongly disagree" to "strongly agree" beneath two statements: "If this man (I) got in a fistfight with an average male undergraduate student, this man (I) would probably win." The difference between self- and targetrated dominance was used to calculate a "relative dominance" variable for each participant (see Puts et al. 2006).

\section{Acoustic Analyses}

Mean $F_{0}, F_{0}$ variation (operationalized as the within-subject standard deviation of $F_{0}$, hereafter called $F_{0}$-SD), utterance duration (seconds), intensity (decibels, dB), and formant dispersion $\left(D_{f}\right)$ were measured using Praat voice analysis software (version 4.4.11) for each of the participants' three recordings (control, courtship, competitive). All parameters were set to the programmers' recommendations, including a pitch floor of $75 \mathrm{~Hz}$ and a pitch ceiling of $300 \mathrm{~Hz}$. For characteristics of these recordings see Table 1. $D_{f}$ was obtained by the following methods 
Table 1 Descriptive statistics

\begin{tabular}{llll}
\hline Vocal Parameters & Control & Courtship & Competitive \\
\hline Mean $F_{0}(\mathrm{SD})$ & $112.6 \mathrm{~Hz}(15.1)$ & $112.7 \mathrm{~Hz}(15.1)$ & $113.2 \mathrm{~Hz}(14.5)$ \\
$F_{0}$ Range & $85.7-136.5 \mathrm{~Hz}$ & $82.9-158.9 \mathrm{~Hz}$ & $85.6-154.6 \mathrm{~Hz}$ \\
$F_{0}$-SD (SD) & $12.9 \mathrm{~Hz}(4.1)$ & $11.6 \mathrm{~Hz}(3.9)$ & $12.3 \mathrm{~Hz}(4.5)$ \\
Mean Duration (SD) & $28.8 \mathrm{~s}(4.6)$ & $20.5 \mathrm{~s}(9.3)$ & $17.9 \mathrm{~s}(6.5)$ \\
Mean Intensity (SD) & $68.9 \mathrm{~dB}(4.1)$ & $60.3 \mathrm{~dB}(4.3)$ & $61.2 \mathrm{~dB}(4.6)$ \\
Mean $D_{f}(\mathrm{SD})$ & $938 \mathrm{~Hz}(60)$ & $936.7 \mathrm{~Hz}(64)$ & $936.9 \mathrm{~Hz}(64)$ \\
\hline
\end{tabular}

$F_{0}-\mathrm{SD}=F_{0}$ variation, $D_{f}=$ Formant dispersion

(Gonzalez 2004; Xue and Hao 2003): Spectral information was obtained using the long-term average spectrum (LTAS), a fast Fourier transform (FFT)-generated frequency power spectrum represented in a running speech acoustic signal. (Voices were first resampled at $11025 \mathrm{~Hz}$; otherwise, all values were set to Praat's authors' recommendations.) An LPC smoothing analysis generated a smooth curve from the LTAS, with six peaks and a pre-emphasis coefficient of 0.5 . Using edit and draw tools, we confirmed that the LPC curve matched well to the LTAS. When a six-peak model did not conform well to the LTAS, seven peaks were used. Formant values $(F 1-F 4)$ were taken from the first four peaks of the LPC curve; $D_{f}$ was calculated by computing the average distance between each of the four peaks (Fitch 1997). All formants were analyzed a second time using the same procedures in order to obtain test-retest reliability. Correlations between first and second measurements were high owing to the semi-automated nature of the method; for all three recordings, $r=0.99$. $D_{f}$ values used in all analyses were the averages of the first and second measurements.

\section{Ratings of Dominance by Men}

Competitive recordings were used as stimuli for male "audio raters." Raters listened to a set of 30 or 31 recordings, which included a random selection of raised, lowered, and unmanipulated recordings (10 or 11 of each). Importantly, only unmanipulated recordings were included in analyses for the present study. No set contained two recordings of the same dating-game participant. Recordings were rated for perceived social and physical dominance using the procedures described above. Mean physical and social dominance scores were created for each of the voices $(N=111)$ by averaging across the raters' responses (mean raters/recording $=8)$.

Transcribed competitive recordings were read by male "content raters." Each rater read a random selection of 28 to 31 transcribed recordings and rated each transcript for perceived social and physical dominance, mirroring procedures for audio recordings.

\section{Ratings of Attractiveness by Women}

Female "audio raters" listened to a selection of 30 or 31 courtship recordings (analogous to procedures described above) and rated each target's attractiveness for a 
"short-term, purely sexual relationship, such as a one-night stand," and then for a "long-term, committed relationship." Female raters were told to place a mark on a visual analogue scale anchored at "extremely unattractive" and "extremely attractive." Mean short-term and long-term attractiveness scores were created for each of the voices $(N=111)$ by averaging across the fertile (mean raters/recording= 3.5 ) and non-fertile (mean raters/recording $=9$ ) raters' responses.

Transcribed courtship recordings were read by female "content raters." Each rater read a random selection of 28 to 31 transcribed recordings and rated each transcript for short-term or long-term attractiveness, mirroring procedures described above.

\section{Menstrual Cycle Classification}

Menstrual cycle information was collected from all female audio and content raters through self report. The participants were asked to circle the number of days in their typical menstrual cycle ( $<22$ to $>38 ; M=29.2$ days) and the date of onset of their last menstrual bleeding on a calendar. Each rater was categorized as being in either the "fertile" (audio: $N=38$, content: $N=17$ ) or "non-fertile" (audio: $N=98$, content: $N=18$ ) phase of her cycle, according to a conservative eight-day window of fertility beginning six days before the presumptive day of ovulation and ending one day after (see Puts 2005 for further methodological details). Raters were neither pregnant nor using hormonal contraceptives at the time of the study. Menstrual-cycle effects are not a central focus of this study, but because effects have previously been observed in studies of voice judgments, we attempted to monitor such variation.

\section{Results}

Relationships between Predictors

$F_{0}$ and $F_{0}$-SD were correlated in each of the three recording types: control $(r=0.43$, $p<0.001)$, courtship $(r=0.44, p<0.001)$, and competitive $(r=0.35, p<0.001)$. Both $F_{0}$ and $F_{0}$-SD changed between the control and the two experimental conditions, and the change in $F_{0}$ was significantly correlated with the change in $F_{0}$-SD for both competitive $(r=0.43, p<0.001)$ and courtship $(r=0.43, p<0.001)$ recordings. Within participants, $F_{0}$ and $F_{0}$-SD were correlated with themselves across each of the three recordings (mean $F_{0}$ : average $r=0.92$, and $F_{0}$-SD: average $r=0.50$ ).

$D_{f}$ was correlated with $F_{0}$-SD only in the control recording $(r=0.26, p<0.01)$ and was not correlated with $F_{0}$ for any of the three recording types. $D_{f}$ did not change significantly from control to either courtship $\left(t_{109}=0.20\right.$, n.s. $)$ or competitive $\left(t_{109}=0.15\right.$, n.s. $)$ recordings.

Intensity was positively correlated with mean $F_{0}$ for both competitive $(r=0.31$, $p<0.01)$ and courtship $(r=0.26, p<0.01)$ recordings.

\section{Zero-order Correlations}

Vocal physical dominance was significantly correlated with $F_{0^{-}}$SD $(r=-0.29$, $p<0.01)$ and content physical dominance $(r=0.65, p<0.001)$. Vocal social 
dominance was significantly correlated with $F_{0}$-SD $(r=-0.21, p<0.05)$, intensity $(r=0.19, p<0.05)$, and content social dominance $(r=0.60, p<0.001)$. (See Table 2 for marginal and nonsignificant correlations.)

Vocal short-term attractiveness ratings by fertile-phase females were correlated with mean $F_{0}(r=-0.31, p<0.001), F_{0}$ SD $(r=-0.24, p<0.01)$, long-term vocal attractiveness ratings $(r=0.77, p<0.001)$, and short-term content attractiveness ratings $(r=0.27, p<0.01)$. Vocal short-term attractiveness ratings by non-fertilephase females were correlated with mean $F_{0}(r=-0.24, p<0.001), F_{0}$-SD $(r=-0.25$, $p<0.01)$, long-term vocal attractiveness ratings $(r=0.69, p<0.001)$, and short-term content attractiveness ratings $(r=0.27, p<0.01)$.

Vocal long-term attractiveness ratings by fertile-phase females were correlated with mean $F_{0}(r=-0.32, p<0.001), F_{0}$ SD $(r=-0.29, p<0.01)$, intensity $(r=0.22$, $p<0.05)$, and long-term content attractiveness ratings $(r=0.26, p<0.01)$. Vocal longterm attractiveness ratings by non-fertile-phase females were correlated with mean $F_{0}(r=-0.22, p<0.001)$, intensity $(r=-0.22, p<0.05)$, and long-term content attractiveness ratings $(r=0.34, p<0.001)$. (See Table 3 for marginal and nonsignificant correlations.)

\section{Changes in $F_{0}$ Variation $\left(F_{0}-\mathrm{SD}\right)$ across Conditions}

There was a significant decrease in $F_{0}$-SD from control $(M=12.9)$ to courtship recording $\left(M=11.6\right.$; two-tailed paired $t$-test: $\left.t_{110}=3.76, p<0.001\right)$. Participants also reduced their $F_{0}$-SD from control to competitive $(M=12.3)$, but the difference was not statistically significant $\left(t_{110}=1.54, p=0.13\right)$. Additionally, changes in $F_{0}$-SD from control to courtship varied significantly with participants' perceptions of their relative physical dominance $(r=-0.23, p=0.02)$ : males who rated themselves as

Table 2 Correlations among variables: competitive recording

\begin{tabular}{|c|c|c|c|c|c|c|c|c|}
\hline & $\begin{array}{l}\text { Content } \\
\text { Physical } \\
\text { Dominance }\end{array}$ & $\begin{array}{l}\text { Vocal } \\
\text { Social } \\
\text { Dominance }\end{array}$ & $\begin{array}{l}\text { Content } \\
\text { Social } \\
\text { Dominance }\end{array}$ & Mean $F_{0}$ & $F_{0}-\mathrm{SD}$ & $D_{f}$ & Intensity & Duration \\
\hline $\begin{array}{c}\text { Vocal Physical } \\
\text { Dominance }\end{array}$ & $.65 * * * *$ & $.73 * * * *$ & $.40 * * * *$ & $-.18 *$ & $-.29 * * *$ & .03 & .07 & $.18^{*}$ \\
\hline $\begin{array}{l}\text { Content Physical } \\
\text { Dominance }\end{array}$ & - & $.54 * * * *$ & $.44 * * * *$ & -.02 & $-.17^{*}$ & .10 & .08 & .14 \\
\hline $\begin{array}{l}\text { Vocal Social } \\
\text { Dominance }\end{array}$ & - & - & $.60 * * * *$ & -.15 & $-.21^{* *}$ & .07 & $.19 * *$ & .11 \\
\hline $\begin{array}{l}\text { Content Social } \\
\text { Dominance }\end{array}$ & - & - & - & -.06 & -.15 & $.19 * *$ & $.23 * *$ & $.22 * *$ \\
\hline Mean $F_{0}$ & - & - & - & - & $.35 * * * *$ & -.10 & $.31 * * *$ & -.07 \\
\hline$F_{0}$-SD & - & - & - & - & - & .03 & .10 & .11 \\
\hline$D_{f}$ & - & - & - & - & - & - & .10 & .04 \\
\hline Intensity & - & - & - & - & - & - & - & .02 \\
\hline
\end{tabular}

$F_{0}-\mathrm{SD}=F_{0}$ variation, $D_{f}=$ Formant dispersion

$* p<.10, * * p<.05, * * * p<.01, * * * * p<.001$ 


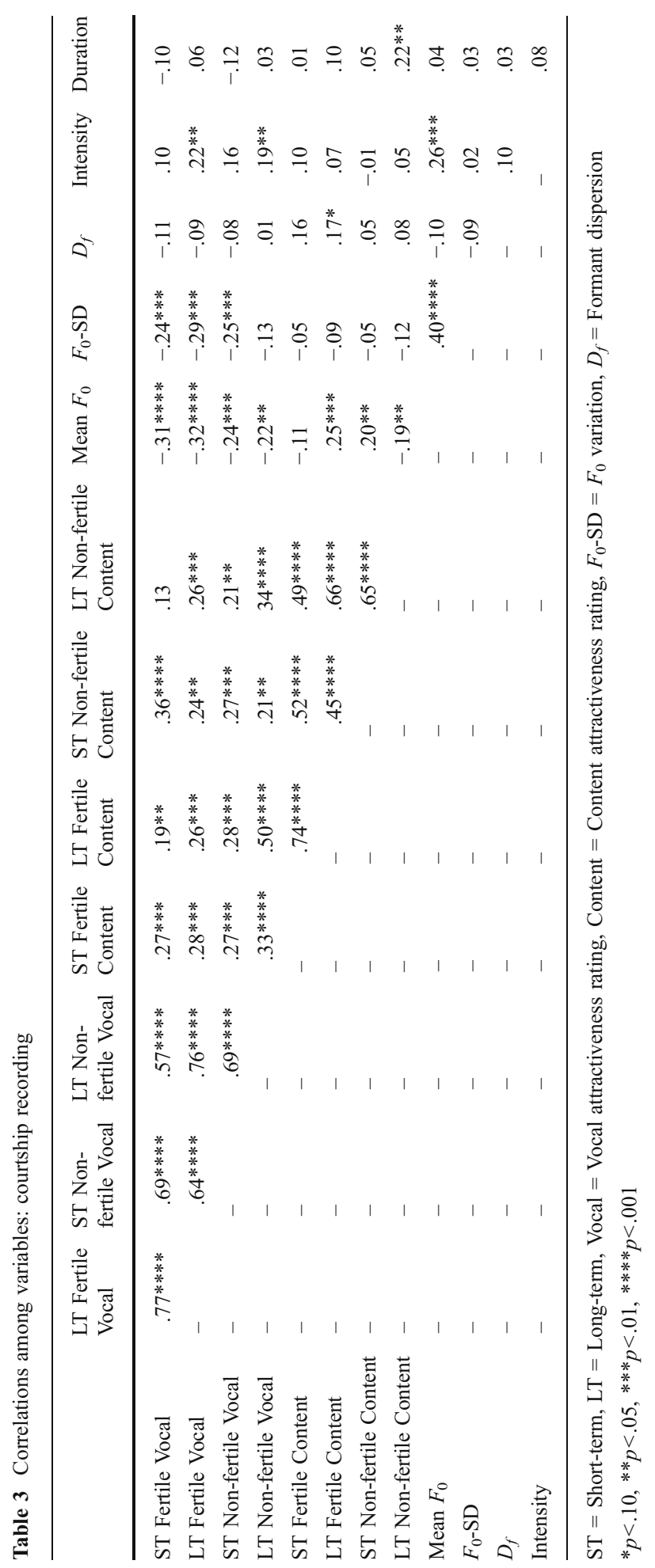


Table 4 Multiple regression predicting ratings of physical and social dominance

\begin{tabular}{lccc}
\hline Predictors & $B$ & SE $B$ & $\beta$ \\
\hline Predicting physical dominance & & & $-.16^{* *}$ \\
$F_{0}$-SD & -0.47 & .23 & $-.14^{*}$ \\
$F_{0}$ Mean & -0.13 & .08 & -.06 \\
$D_{f}$ & -0.01 & .01 & .09 \\
Duration & 0.19 & .15 & .08 \\
Intensity & 0.24 & .22 & $.62^{* * * *}$ \\
Content (physical) & 8.48 & 1.01 & -.09 \\
Predicting social dominance & & & -.14 \\
$F_{0}$-SD & -0.27 & .27 & -.06 \\
$F_{0}$ Mean & -0.13 & .09 & -.02 \\
$D_{f}$ & -0.01 & .02 & .11 \\
Duration & -0.04 & .17 & $.56^{* * * *}$ \\
Intensity & 0.34 & .26 & 1.53 \\
Content (social) & 10.05 & & \\
\hline
\end{tabular}

Mean and standard deviation from competitive recording

$F_{0}-\mathrm{SD}=F_{0}$ variation, $D_{f}=$ Formant dispersion

$* p<.10, * * p<.05, * * * p<.01, * * * * p<.001$

more physically dominant tended to decrease $F_{0}$-SD to a greater degree. Changes in $F_{0}$-SD from control to competitive did not vary significantly with relative dominance $(r=-0.12, p=0.26)$. Puts et al. (2006) reported that changes in mean $F_{0}$ from control to competitive varied with perceptions of relative physical dominance. Controlling for $F_{0}$-SD does not change this result (partial $r=-0.24, p=0.01$ ).

Predicting Judgments of Physical and Social Dominance by Men

Acoustic and content variables were entered into multiple regression models to predict attractiveness and dominance ratings. Given that the predictor variables were often correlated, multicollinearity was a concern. Consequently, variance inflation factors (VIFs) were assessed in order to test for multicollinearity in all multiple regression models. In all models, VIFs were $<1.3$ for each of the predictors, and therefore the results of these multiple regression analyses are unlikely to be confounded by multicollinearity.

Because $F_{0}$ and $F_{0}$-SD are positively correlated (Tables 2 and 3), it is possible that previously reported correlations concerning the relationship between $F_{0}$ and dominance (or attractiveness) are due instead to the influence of $F_{0}-\mathrm{SD}$. In order to assess the independent effects of mean $F_{0}$ and $F_{0}$-SD, both variables were entered simultaneously along with mean duration, intensity, $D_{f}$, and mean content rating into separate multiple regression models for each of the two dominance variables (see Table 4$)$. These models explained 49.7\% $\left(R=0.705, F_{6,101}=16.63\right.$, $p<0.001)$ of the variance in physical dominance ratings and $39 \%\left(R=0.625, F_{6,101}=\right.$ $10.77, p<0.001)$ of the variance in social dominance ratings. $F_{0}$-SD significantly 
Table 5 Multiple regression predicting judgments of short-term and long-term attractiveness by menstrual cycle phase

\begin{tabular}{|c|c|c|c|c|c|c|}
\hline \multirow[b]{2}{*}{ Predictors } & \multicolumn{3}{|c|}{ Fertile-phase women } & \multicolumn{3}{|c|}{ Non-fertile-phase women } \\
\hline & $B$ & SE $B$ & $\beta$ & $B$ & SE $B$ & $\beta$ \\
\hline \multicolumn{7}{|c|}{ Predicting short-term attractiveness ratings } \\
\hline$F_{0}-\mathrm{SD}$ & -0.66 & .45 & -.15 & -0.83 & .39 & $-.21 * *$ \\
\hline$F_{0}$ Mean & -0.32 & .12 & $-.27 * * *$ & -0.17 & .10 & $-.17 *$ \\
\hline$D_{f}$ & -0.05 & .02 & $-.19 * *$ & -0.03 & .02 & -.13 \\
\hline Duration & -0.13 & .16 & -.07 & -0.18 & .14 & -.10 \\
\hline Intensity & 0.89 & .40 & $.20 * *$ & 0.89 & .32 & $.25 * * *$ \\
\hline Content & 6.16 & 2.25 & $.25 * * *$ & 5.05 & 1.87 & $.24 * * *$ \\
\hline \multicolumn{7}{|c|}{ Predicting long-term attractiveness ratings } \\
\hline$F_{0}-\mathrm{SD}$ & -0.92 & .44 & $-.20 * *$ & -0.24 & .41 & -.06 \\
\hline$F_{0}$ Mean & -0.28 & .12 & $-.24 * *$ & -0.23 & .11 & $-.21 * *$ \\
\hline$D_{f}$ & -0.04 & .02 & $-.17 * *$ & 0.01 & .02 & -.06 \\
\hline Duration & 0.12 & .16 & .06 & -0.09 & .16 & -.05 \\
\hline Intensity & 1.16 & .39 & $.27 * * *$ & 0.94 & .39 & $.26 * * *$ \\
\hline Content & 5.27 & 1.86 & $.26 * * *$ & 5.26 & 1.64 & $.30 * * *$ \\
\hline
\end{tabular}

Mean and standard deviation from courtship recording.

$F_{0}$-SD $=F_{0}$ variation, $D_{f}=$ Formant dispersion

$* p<.10, * * p<.05, * * * p<.01$

predicted judgments of physical dominance $(\beta=-0.16, p<0.05)$, and mean $F_{0}$ approached significance as a predictor $(\beta=-0.14, p=0.08)$. Neither $F_{0}$-SD $(\beta=-0.09$, n.s. $)$ nor mean $F_{0}(\beta=-0.14$, n.s. $)$ explained a significant portion of the variance in judgments of social dominance. $D_{f}$ also did not capture any additional variance in physical $(\beta=-0.06$, n.s.) or social $(\beta=-0.06$, n.s.) dominance ratings; nor did intensity (physical: $\beta=0.08$, n.s., social: $\beta=0.11$, n.s.). Dominant content ratings were a strong positive predictor of both physical $(\beta=0.62, p<0.001)$ and $\operatorname{social}(\beta=0.56, p<0.001)$ dominance ratings.

In order to control for possible interactions between acoustic parameters and content, we conducted exploratory stepwise multiple regressions to examine which variables would explain significant proportions of the variation in ratings when all variables and their interactions were included in the analyses. Vocal parameters, content, and the interaction terms (the products of each of the vocal parameters with content) were entered into a stepwise multiple regression to predict physical and social dominance.

The relationships between vocal parameters and perceptions of physical dominance were relatively unchanged by these controls: only $F_{0}$-SD and physically dominant content were significant predictor variables $(\beta=-0.18, p=0.011$ and $\beta=0.62, p<0.001$, respectively). The interaction between $D_{f}$ and content $(\beta=-0.16$, $p=0.021$ ) also significantly predicted physical dominance. To explore this interaction, we regressed physical dominance on $D_{f}$ at one standard deviation above 
and at one standard deviation below the mean on physically dominant linguistic content. There was a marginally significant inverse relationship between physical dominance and $D_{f}$ for those with highly dominant content only $(\beta=-0.20, p=0.055)$. In other words, a low, masculine $D_{f}$ predicted increased ratings of physical dominance more for men who used physically dominant language.

For perceptions of social dominance, the effects were similar to the initial model: only socially dominant content was a significant predictor variable $(\beta=0.52$, $p<0.001)$. The interaction between duration and content $(\beta=-0.25, p<0.01)$ was also a significant predictor. An analysis of the simple slopes revealed a significant positive relationship between duration and social dominance for those with higher socially dominant content $(\beta=0.35, p<0.05)$, but the inverse for those with lower socially dominant content $(\beta=-0.28, p<0.05)$. That is, speaking for a longer time increased social dominance for those who spoke with socially dominant language but decreased dominance ratings for those who did not use dominant-sounding language. In addition, the interaction between intensity and content $(\beta=-0.15$, $p=0.04$ ) achieved significance. There was a significant positive relationship between social dominance and intensity for those with low socially dominant content only $(\beta=0.25, p<0.05)$. In other words, for men who used language that was perceived as lacking in social dominance, speaking more loudly increased their social dominance ratings.

For neither physical nor social dominance were there significant interactions between speech content and either mean $F_{0}$ or $F_{0}-\mathrm{SD}$.

\section{Predicting Judgments of Attractiveness by Women}

Multiple regression models were used to assess the impact of vocal parameters on women's attractiveness judgments. These models explained $25.3 \%\left(R=0.503, F_{6,101}\right.$ $=5.52, p<0.001)$ and $29 \%\left(R=0.538, F_{6,98}=6.67, p<0.001\right)$ of the variance in fertile-phase women's judgments of short- and long-term attractiveness, respectively, and $23.2 \%\left(R=0.428, F_{6,101}=5.10, p<0.001\right)$ and $20.4 \%\left(R=0.451, F_{6,101}=4.30\right.$, $p<0.01)$ for non-fertile women's judgments. For the case most revealing of goodgenes mate choice, fertile-phase women judging short-term attractiveness, mean $F_{0}$ achieved significance $(\beta=-0.27, p<0.01)$ whereas $F_{0}$-SD did not $(\beta=-0.15$, n.s. $)$. Mean $F_{0}$ also predicted judgments of short- and long-term attractiveness by fertilephase women $(\beta=-0.27, p<0.01$ and $\beta=-0.24, p<0.05$, respectively) and nonfertile women $(\beta=-0.17, p<0.10$ and $\beta=-0.21, p<0.05)$. Results were inconsistent for $F_{0}-\mathrm{SD}$, which predicted long-term attractiveness judgments by fertile $(\beta=-0.20, p<0.05)$, but not non-fertile $(\beta=-0.06$, n.s. $)$, women, and short-term attractiveness judgments by non-fertile $(\beta=-0.21, p<0.05)$, but not fertile $(\beta=$ -0.15 , n.s.), women. $D_{f}$ was a significant predictor of short-term and long-term attractiveness judgments by fertile $(\beta=-0.19, p<0.05$ and $\beta=-0.17, p<0.05)$, but not by non-fertile $(\beta=-0.13$, n.s. and $\beta=-0.06$, n.s. $)$, women. Both intensity and content ratings also positively predicted attractiveness across menstrual phases and mating contexts $(\beta$-values $\geq 0.20, p$-values $<0.05)$. (See Table 5.)

Stepwise multiple regression analyses were conducted, as described above, for short- and long-term attractiveness for both fertile and non-fertile women. Vocal parameters, attractive content, and the interaction terms (the product of each of the 
vocal parameters with attractive content) were entered into stepwise multiple regressions predicting attractiveness judgments across cycle phase and mating context.

Mean $F_{0}(\beta=-0.33, p<0.01)$, intensity $(\beta=0.22, p=0.02)$, and attractive content $(\beta=0.21, p<0.02)$ significantly predicted short-term attractiveness ratings by fertilephase women, whereas mean $F_{0}(\beta=-0.26, p<0.01)$ and attractive content $(\beta=0.26$, $p<0.01)$ significantly predicted short-term ratings by non-fertile women. Mean $F_{0}$ $(\beta=-0.36, p<0.001)$, intensity $(\beta=0.27, p<0.01)$, and attractive content $(\beta=0.21$, $p=0.02)$ significantly predicted long-term attractiveness ratings by fertile-phase women. Similarly, mean $F_{0}(\beta=-0.22, p=0.02)$, intensity $(\beta=0.23, p=0.01)$, and attractive content $(\beta=0.31, p<0.01)$ significantly predicted long-term attractiveness ratings by non-fertile women. In addition, the interaction between mean $F_{0}$ and longterm attractive content achieved significance $(\beta=-0.22, p=0.01)$. Low mean $F_{0}$ increased long-term attractiveness to non-fertile women only for those men with highly attractive content $(\beta=-0.39, p<0.01)$. There were no significant interactions for either fertile-phase women or short-term mating contexts. Again, there was no significant interaction between speech content and either mean $F_{0}$ or $F_{0^{-}}$SD.

\section{Discussion}

The present study indicates that multiple acoustic parameters vary in natural competitive- and courtship-generated men's speech, and that variance in these parameters may be differentially attended to by men evaluating dominance and women evaluating attractiveness. Separate evolved priorities may account for the differences between men's and women's judgments.

We present several novel findings on the role of voice in male-male competition and female mate choice. First, lower $F_{0}$ variation in men's voices predicted increased perceptions of physical dominance in male listeners. Second, $F_{0}$ variation captured some variation in attractiveness judgments by women, but results were inconsistent. Our results suggest that mean $F_{0}$ and $F_{0}$ variation have independent effects, and that they are attended to differently by men and women judging physical dominance and sexual attractiveness, respectively. The role of $F_{0}$ variation is likely to be an important consideration in future research on the social ecology of the human voice. Third, women found speech intensity (i.e., volume) attractive across mating context and menstrual phase. Fourth, semantic content strongly predicted dominance and attractiveness assessments. To our knowledge, this is the first study to examine the associations between perceptions of naturally occurring competitive and courtship interactions, their semantic content, and acoustic parameters.

Formant Dispersion

Although previous experimental research has shown that low $D_{f}$ is perceived as dominant by men (Feinberg et al. 2005; Puts et al. 2007; Wolff and Puts 2010), this was not the case in the present study. A possible explanation is that the variance in $D_{f}$ in this sample was smaller than the manipulations in experimental studies. However, in the control condition, one standard deviation of $D_{f}$ was $60 \mathrm{~Hz}$, 
representing a $6 \%$ deviation from the mean, and the increment of manipulation in Puts et al. (2007) was also $6 \%$. Another possible explanation is that the effects of $D_{f}$ may be small relative to the effects of other acoustic parameters and content that varied freely in the present study. Too little variance in dominance judgments may have remained unexplained by content and these other acoustic parameters for $D_{f}$ to explain a significant portion. Finally, although we used only ratings and measurements of unmanipulated recordings in all analyses, participants also heard recordings raised and lowered in both $F_{0}$ and $D_{f}$. This may have damped the effects of $F_{0}$ and $D_{f}$ on ratings of unmanipulated recordings. While some caution in interpreting the relative effects of these acoustic variables within rating contexts (e.g., physical dominance) is needed, our main findings that acoustic variables differentially predicted ratings between contexts (e.g., attractiveness to women vs. dominance to men) are not undermined.

We found that women in the fertile phase of their cycle attended to low $D_{f}$ when rating short- and long-term attractiveness. Feinberg et al. (2005) found no effect of $D_{f}$ on attractiveness, although manipulations of $D_{f}$ did influence perceptions of age, size, and masculinity-qualities that influence attractiveness to females (e.g., Pawlowski and Jasienska 2005). $D_{f}$ is thought to be an index of vocal tract length, which goes through a secondary growth spurt in males during puberty (Fitch and Giedd 1999), putatively under the influence of circulating androgens. Therefore, like mean $F_{0}$, low $D_{f}$ may indicate genetic quality through its association with immunocompetence - an idea that has not yet been explored in the literature.

\section{Fundamental Frequency (Mean $F_{0}$ )}

Previous studies have shown that lower mean voice pitch increases assessments of some proxies of relative dominance (i.e., height, weight, and age; Collins 2000; Feinberg et al. 2005; Lass and Brown 1978), as well as subjective judgments of physical dominance (Puts et al. 2006, 2007; Wolff and Puts 2010). Although mean $F_{0}$ and both measures of dominance were correlated, when entered into a multiple regression with other voice parameters, mean $F_{0}$ failed to achieve statistical significance.

In contrast, mean $F_{0}$ was a significant predictor of women's assessments of attractiveness. In a previous study using the same voice recordings, Puts (2005) found a significant negative correlation between mean $F_{0}$ and women's attractiveness ratings. In contrast with men's judgments of dominance (where inclusion of $F_{0}$ variation, $D_{f}$, intensity, and duration washes out the effects of mean $F_{0}$ ), women's judgments of attractiveness remain significantly related to $F_{0}$ in a parallel multivariate analysis. Mean $F_{0}$ predicted judgments of attractiveness across cycle phase and relationship context. However, the relationship between mean $F_{0}$ and attractiveness was strongest for fertile-phase women rating short-term attractiveness, suggesting an elevated attention to signals of quality when conception risk is greatest (Gangestad and Thornhill 1998; Penton-Voak et al. 1999; Puts 2005).

\section{$F_{0}$ Variation}

Results showed that male voices low in $F_{0}$ variation were perceived to be physically dominant. In many previous studies of mean $F_{0}, F_{0}$ variation was held constant or 
ignored. In this study we found that both mean $F_{0}$ and $F_{0}$-SD vary naturally in unmanipulated utterances, and that the two are moderately correlated. By controlling each of these parameters using multiple regression, we found evidence that men attend strongly to $F_{0}$ variation when making physical dominance attributions.

One proximate mechanism that may clarify the relationship between low $F_{0}$ variation and perceptions of high physical dominance is autonomic arousal. Both mean $F_{0}$ and $F_{0}$ variation are increased during states of heightened autonomic arousal. The vocal characteristics of high-activation emotions (e.g., panic fear and "hot" anger) include high mean $F_{0}$ and high $F_{0}$ variation (Banse and Scherer 1996). $F_{0}$ increases as a result of greater tension on the vocal folds (Titze 2000), which may also increase $F_{0}$ variation owing to the loss of fine motor control (Goedeking 1988).

On an ultimate level, indicators of arousal may be important signals in dominance contexts. The primary function of autonomic activation in antagonistic interactions is to prepare the body for fighting or fleeing. This physiological preparation affects the laryngeal muscles, which in turn affect voice characteristics (Scherer 1986). Perhaps only those who are assured of their own size, strength, or ability can afford not to prepare for a costly fight or a quick flight. In this way, lack of arousal may function as a costly signal (Scherer 1986; Zahavi 1982). Thus, vocal signals associated with lack of arousal- low $_{F_{0}}$ variation and low $F_{0}$-may indicate the signaler's self perceptions of relative dominance when facing a challenge.

Constraints of the vocal apparatus may ensure that the signal remains honest and uncorrupted by cheaters. Because the vocal system is not a distinct system but rather is overlaid on more primary structures (e.g., those of respiration and digestion; Johnstone and Scherer 2005; Scherer 1986), and because the qualities of vocalizations are affected by states of the body via its connection with the lungs and diaphragm (Titze 1994), it may be difficult to alter particular vocal signals without altering more basic functioning (e.g., breathing), and vice versa. This functional linkage may account for men's selective attention to individual differences in $F_{0}$ variation.

Mean $F_{0}$ is associated with both the signaler's vocal fold size and state of arousal. However, $F_{0}$ variation is not as confounded by individual differences in vocal fold morphology and may therefore provide a more pure signal of arousal. These dynamics would apply not only to male dominance contests but to competitive interactions among females as well.

The hypothesized link between $F_{0}$ variation and arousal in dominance interactions appears to mesh with what is known about $F_{0}$ variation and friendly interactions. Trainor et al. (2000) proposed that the exaggerated $F_{0}$ contours indicative of infantdirected speech is highly "emotional" speech, which is not categorically different from adult-directed emotional speech. In this sense, $F_{0}$ variation might be the vocal equivalent of a smile - a friendly signal that may have phylogenetic roots in a submissive gesture (Darwin 1872; Ketelaar et al. 2005). The present findings suggest the converse of this relationship: lack of variation may communicate unfriendly or aggressive intentions.

In contrast to findings with male raters, the relationship between women's attractiveness judgments and $F_{0}$ variation was inconsistent; $F_{0}$ variation predicted 
attractiveness for non-fertile females rating short-term attractiveness and fertile females rating long-term attractiveness. Several studies have found that moderate to high variation in $F_{0}$ is most "attractive" to people in general (Ray et al. 1991; Zuckerman and Miyake 1993); however, these studies fail to take into account functional specificity in attractiveness criteria based on evolutionary considerations (i.e., what is physically attractive may depend on the sex of the target and on whether one is discriminating among potential mates or potential allies; Tooby and Cosmides 1992). Riding et al. (2006) found a decrease in social attractiveness in men's voices with increasing $F_{0}$ variation; however, the effect was not significant. Clearly, more research is needed to determine whether factors such as individual differences or contextual variation can account for inconsistent findings on the perceived attractiveness of $F_{0}$ variation.

Previous research has found that preferences for traits theoretically associated with high genetic quality peak during the fertile phase of the ovulatory cycles and/or when evaluating short-term mates (e.g., Gangestad and Thornhill 1998; Penton-Voak et al. 1999; Puts 2005). However, unlike mean $F_{0}, F_{0}$ variation appears to exhibit low heritability (Debruyne et al. 2002) and thus may be a poor indicator of genetic quality. If $F_{0}$ variation does not primarily signal genetic quality but rather indicates state-dependent changes in arousal, then increased attention to $F_{0}$ variation during ovulation or in short-term mating contexts would not necessarily be a good design for sire recruitment. In accordance with this reasoning, we did not find consistent attention to $F_{0}$ variation in these contexts.

Recent research suggests that the evolutionary effects of female choice in humans may be overestimated in part because a number of traits widely assumed to be attractive to females actually have a greater impact on male dominance (Puts 2010). In parallel with this perspective, we recently showed that, in a sample of college-age men, $F_{0}$ variation is a better predictor of male mating success than is $F_{0}$ (Hodges-Simeon et al. 2010).

The results reported here may indicate functional specificity in attention to features of voice (Searcy and Nowicki 2005). When judging attractiveness, women may respond to features of the voice that are indicative of heritable mate quality (i.e., "good genes") - low mean $F_{0}$ and $D_{f}$. Among men, the functionally important signal components for intrasexual competitors may be twofold: previous research has shown that signals of static quality or formidability are important; however, competitors must also attend to signal features (i.e., $F_{0}$ variation) that indicate more state-dependent qualities, such as arousal.

The present findings also highlight some differences between physical and social dominance. Although the two are overlapping constructs, physical dominance (as rated by men) was related to low $F_{0}$ variation, but social dominance was not. In humans, there may be multiple ways to attain status (Henrich and Gil-White 2001). Physical dominance was defined for participants in the present study as the ability to win a physical fight. Social dominance, in contrast, may also require effective communication and leadership. Lack of sufficient pitch variation might impair one's ability to achieve these goals, and therefore the ability to win friends, allies, and supporters. $F_{0}$ variation in this sense quantifies the zero-sum trade-off between signaling affiliation and aggression. $F_{0}$ variation across differing 
contexts may be used to assay the balance of these motivational states in specific social interactions.

\section{Intensity}

Another interesting result was a set of positive relationships between voice intensity and all measures of vocal attractiveness by women. A possible explanation is that voice intensity in men reflects vigor and is analogous to roaring rate in red deer stags, which predicts actual fighting success (Clutton-Brock and Albon 1979) and appears to positively influence attractiveness to females (McComb 1991). However, this interpretation seems contradicted by the lack of statistically significant relationships between intensity and either dominance rating. The role of voice intensity in human female choice and male contests warrants further investigation. In addition, the correlation between intensity and mean $F_{0}$ suggests the importance of measuring and controlling for variation in intensity when studying the effect of mean $F_{0}$ on interpersonal perceptions.

\section{Changes in $F_{0}$ Variation across Conditions}

State-dependent changes may be ascertained by examining changes in $F_{0}$ variation from one condition to another. Results indicated that $F_{0}$ variation decreased from control to courtship and that this change was associated with the participants' perceptions of their own physical dominance; participants who rated themselves as more physically dominant relative to their competitor tended to lower $F_{0}$ variation to a greater degree when speaking to the woman. Signaling low arousal via low $F_{0}$ variation is potentially important to perceptions of both dominance and attractiveness, as our results show. Thus, our male participants might generally have been motivated to lower $F_{0}$ variation during mating competition. However, physically dominant men were apparently more successful at this, perhaps because they were more confident of their success and therefore less nervous. Although participants also tended to decrease $F_{0}$ variation from control to competitive condition, this difference was not significant. For a discussion of changes in mean $F_{0}$ across conditions, see Puts et al. (2006).

\section{Verbal Content}

Verbal content was a strong predictor of both dominance judgments by men and attractiveness judgments by women. Although verbal content per se is not the focus of this paper, it should come as no surprise that men rated highly on physical dominance used such phrases as "I'm a lot bigger and stronger than most men." Men rated highly on social dominance often referred to holding office in a fraternity. The male most attractive to female raters spoke of being an athlete; being tall and physically fit; liking to go out, watch movies, and hang out; as well as having a good sense of humor. We also examined content by acoustic parameter interactions in order to determine whether content moderates perceptions of different vocal characteristics (Jones et al. 2008). Overall, there were very few interactions and no interactions between content and either $F_{0}$ or $F_{0}$-SD. 


\section{Limitations and Conclusions}

Acoustic parameters and verbal content explained $20-50 \%$ of the variance in perceptions of dominance and attractiveness. Although a variety of idiosyncratic preferences could account for the amount of variance unexplained, there may be other acoustic factors that systematically affect participants' perceptions of dominance and attractiveness.

Dominance and attractiveness assessments depend on a variety of factors in multiple sensory modalities (Searcy and Nowicki 2005). We explored the potential effects of natural variation in multiple voice parameters on such assessments in unscripted speech samples from an ecologically valid competitive interaction, statistically controlling potential confounding variables via multiple regression. The present research suggests that multiple vocal parameters affect dominance and attractiveness judgments among humans, and that these acoustic variables may be weighted differently by men and women as a function of their different evolved priorities.

Acknowledgments We thank Lisa Brevard, Jerome Lee, Rebecca Prosser, John Putz, Melinda Putz, and Linda Snyder for their conscientious assistance in study preparation and data collection; Julio Gonzalez and Drew Rendall for their advice on measuring formant frequencies; Katherine Verdolini Abbott for providing research support; and Jane Lancaster, Nathan Pipitone, and two anonymous reviewers for their helpful comments on a previous draft of this paper.

Open Access This article is distributed under the terms of the Creative Commons Attribution Noncommercial License which permits any noncommercial use, distribution, and reproduction in any medium, provided the original author(s) and source are credited.

\section{References}

Apicella, C. L., Feinberg, D. R., \& Marlowe, F. W. (2007). Voice pitch predicts reproductive success in male hunter-gatherers. Biology Letters, 3, 682-684.

Archer, J. (2006). Testosterone and human aggression: an evaluation of the challenge hypothesis. Neuroscience and Biobehavioral Reviews, 30, 319-345.

Aronovitch, C. D. (1976). The voice of personality: stereotyped judgments and their relationship to voice quality and sex of speaker. Journal of Social Psychology, 99, 207-220.

Baken, R. J. (1987). Clinical measurement of speech and voice. Boston: College-Hill Press.

Banse, R., \& Scherer, K. R. (1996). Acoustic profiles in vocal emotion expression. Journal of Personality and Social Psychology, 70, 614-636.

Bruckert, L., Lienard, J., Lacroix, A., Kreutzer, M., \& Leboucher, G. (2006). Women use voice parameters to assess men's characteristics. Proceedings of the Royal Society of London, Series B, 273, 83-89.

Clutton-Brock, T. H., \& Albon, S. D. (1979). The roaring of red deer and the evolution of honest advertisement. Behaviour, 69, 145-170.

Collins, S. A. (2000). Men's voices and women's choices. Animal Behaviour, 60, 773-780.

Dabbs, J. M., \& Mallinger, A. (1999). High testosterone levels predict low voice pitch among men. Personality and Individual Differences, 27, 801-804.

Darwin, C. (1872). On the expression of emotions in man and animals (3rd ed.). New York: Oxford University Press. 1998.

Debruyne, F., Decoster, W., Van Gijsel, A., \& Vercammen, J. (2002). Speaking fundamental frequency in monozygotic and dixygotic twins. Journal of Voice, 16, 466-471.

Evans, S., Neave, N., \& Wakelin, D. (2006). Relationships between vocal characteristics and body size and shape in human males: an evolutionary explanation for a deep male voice. Biological Psychology, $72,160-163$.

Evans, S., Neave, N., Wakelin, D., \& Hamilton, C. (2008). The relationship between testosterone and vocal frequencies in human males. Physiology \& Behavior, 93, 783-788. 
Fant, G. (1960). Acoustic theory of speech production. The Hague: Mouton.

Feinberg, D. R. (2008). Are human faces and voices ornaments signaling common underlying cues to mate value? Evolutionary Anthropology, 17, 112-118.

Feinberg, D. R., Jones, B. C., Little, A. C., Burt, D. M., \& Perrett, D. I. (2005). Manipulations of fundamental and formant frequencies influence the attractiveness of human male voices. Animal Behaviour, 69, 561-568.

Feinberg, D. R., Jones, B. C., Law Smith, M. J., Moore, F. R., DeBruine, L. M., Cornwell, R. E., et al. (2006). Menstrual cycle, trait estrogen level, and masculinity preferences in the human voice. Hormones and Behavior, 46, 215-222.

Fitch, W. T. (1997). Vocal tract length and format frequency dispersion correlated with body size in rhesus macaques. Journal of the Acoustical Society of America, 102, 1213-1222.

Fitch, W. T., \& Giedd, J. (1999). Morphology and development of the human vocal tract: a study using magnetic resonance imaging. Journal of the Acoustical Society of America, 106, 1511-1522.

Folstad, I., \& Karter, A. J. (1992). Parasites, bright males and the immuno-competence handicap. American Naturalist, 139, 603-622.

Gangestad, S. W., \& Thornhill, R. (1998). Menstrual cycle variation in women's preferences for the scent of symmetrical men. Proceedings of the Royal Society of London. Series B, 265, 927-933.

Goedeking, P. (1988). Vocal play behavior in cotton-top tamarins. In D. Todt, P. Goedeking, \& D. Symmes (Eds.), Primate vocal communication (pp. 133-144). Berlin: Springer.

Gonzalez, J. (2004). Formant frequencies and body size of speaker: a weak relationship in adult humans. Journal of Phonetics, 32, 277-287.

Grossman, C. J. (1985). Interaction between the gonadal steroids and the immune system. Science, 227, $257-261$.

Hamilton, W. D., \& Zuk, M. (1982). Heritable true fitness and bright birds: a role for parasites? Science, 218, 384-387.

Harries, M. L. L., Walker, J. M., Williams, D. M., Hawkins, S., \& Hughes, I. A. (1997). Changes in the male voice at puberty. Archives of Disease in Childhood, 77, 445-447.

Henrich, J., \& Gil-White, F. (2001). The evolution of prestige: freely conferred deference as a mechanism for enhancing the benefits of cultural transmission. Evolution and Human Behavior, 22, 165-196.

Hodges-Simeon, C. R., Gaulin, S. J. C., \& Puts, D. A. (2010). Voice correlates of mating success in men: examining "contests" versus "mate choice" modes of sexual selection. Archives of Sexual Behavior. doi:10.1007/s10508-010-9625-0.

Hollien, H., Green, R., \& Massey, K. (1994). Longitudinal research on adolescent voice change in males. Journal of the Acoustical Society of America, 96, 2646-2654.

Hughes, S. M., Dispenza, F., \& Gallup, G. G. (2004). Ratings of voice attractiveness predict sexual behavior and body configuration. Evolution and Human Behavior, 25, 295-304.

Johnstone, T., \& Scherer, K. R. (2005). Vocal communication of emotion. In: M. Lewis, \& J. M. HavilandJones (Eds.), Handbook of emotion, (pp. 220-235). Guilford Press.

Jones, B. C., Feinberg, D. R., DeBruine, L. M., Little, A. C., \& Vukovic, J. (2008). Integrating cues of social interest and voice pitch in men's preferences for women's voices. Biology Letters, 4, 192-194.

Jones, B. C., Feinberg, D. R., DeBruine, L. M., Little, A. C., \& Vukovic, J. (2010). A domain-specific opposite-sex bias in human preferences for manipulated voice pitch. Animal Behaviour, 79, 57-62.

Ketelaar, T., Koenig, B., Burkett, B., \& Davis, M. (2005). Money, muscles and smiles: Inferring generosity and physical dominance from smiles. Poster presented at the 18th annual meeting of the Human Behavior and Evolution Society, Philadelphia, PA.

Kimble, C. E., \& Seidel, S. D. (1991). Vocal signs of confidence. Journal of Nonverbal Behavior, 15, 99-105.

Lass, N. J., \& Brown, W. S. (1978). Correlational study of speakers heights, weights, body surface areas and speaking fundamental frequencies. Journal of the Acoustical Society of America, 63, 1218-1220.

Mazur, A., Halpern, C., \& Udry, J. R. (1994). Dominant looking male teenagers copulate earlier. Ethology and Sociobiology, 15, 87-94.

McComb, K. E. (1991). Female choice for high roaring rates in red deer, Cervus elaphus. Animal Behaviour, 41, 79-88.

Morton, E. S. (1977). The occurrence and significance of motivation-structural rules in some bird and mammal species. American Naturalist, 111, 855-869.

Need, A. G., Durbridge, T. C., \& Nordin, B. E. (1993). Anabolic steroids in postmenopausal osteoporosis. Wiener Medizinsche Wochenschrift, 143, 392-395.

Newman, S. R., Butler, J., Hammond, E. H., \& Gray, S. D. (2000). Preliminary report on hormone receptors in the human vocal fold. Journal of Voice, 14, 72-81.

Ohala, J. J. (1983). Cross-language use of pitch: an ethological view. Phonetica, 40, 1-18. 
Pawlowski, B., \& Jasienska, G. (2005). Women's preferences for sexual dimorphism in height depend on menstrual cycle phase and expected duration of relationship. Biological Psychology, 70, 38-43.

Penton-Voak, I. S., Perrett, D., Castles, D., Burt, M., Koyabashi, T., \& Murray, L. K. (1999). Female preferences for male faces change cyclically. Nature, 399, 741-742.

Puts, D. A. (2005). Mating context and menstrual phase affect women's preferences for male voice pitch. Evolution and Human Behavior, 26, 388-397.

Puts, D. A. (2010). Beauty and the beast: mechanisms of sexual selection in humans. Evolution and Human Behavior, 31, 157-175.

Puts, D. A., Gaulin, S. J. C., \& Verdolini, K. (2006). Dominance and the evolution of sexual dimorphism in human voice pitch. Evolution and Human Behavior, 27, 283-296.

Puts, D. A., Hodges, C. R., Cardenas, R. A., \& Gaulin, S. J. C. (2007). Men's voices as dominance signals: vocal fundamental and formant frequencies influence dominance attributions among men. Evolution and Human Behavior, 28, 340-344.

Ray, G. B., Ray, E. B., \& Zahn, C. J. (1991). Speech behavior and social evaluation: an examination of medical messages. Communication Quarterly, 2, 47-57.

Riding, D., Lonsdale, D., \& Brown, B. (2006). The effects of average fundamental frequency and variance of fundamental frequency on male vocal attractiveness to women. Journal of Nonverbal Behavior, 30, $55-61$.

Scherer, K. R. (1986). Vocal affect expression: a review and a model for future research. Psychological Bulletin, 99, 143-165.

Scherer, K. R., London, H., \& Wolf, J. J. (1973). The voice of confidence: paralinguistic cues and audience evaluation. Journal of Research in Personality, 7, 31-44.

Searcy, W. A., \& Nowicki, S. (2005). The evolution of animal communication: reliability and deception in signaling systems. Princeton, NJ: Princeton University Press.

Simpson, J. A., Gangestad, S. W., Christensen, P. N., \& Leck, K. (1999). Fluctuating asymmetry, sociosexuality, and intrasexual competitive tactics. Journal of Personality and Social Psychology, 76, 159-172.

Titze, I. R. (1989). Physiologic and acoustic differences between males and females. Journal of the Acoustical Society of America, 85, 1699-1707.

Titze, I. R. (1994). Principles of voice production. Englewood Cliffs: Prentice Hall.

Titze, I. R. (2000). Principles of voice production (2nd printing). Iowa City: National Center for Voice and Speech.

Tooby, J., \& Cosmides, L. (1992). The psychological foundations of culture. In J. H. Barkow, J. Tooby, \& L. Cosmides (Eds.), The adapted mind (pp. 19-136). New York: Oxford University Press.

Trainor, L. J., Austin, C. M., \& Desjardins, R. M. (2000). Is infant-directed speech prosody a result of the vocal expression of emotion? Psychological Science, 11, 188-195.

van Dommellen, W. A. (1993). Speaker height and weight identification: a reevaluation of some old data. Journal of Phonetics, 21, 337-341.

van Dommellen, W. A., \& Moxness, B. H. (1995). Acoustic parameters in speaker height and weight identification: sex specific behavior. Language and Speech, 38, 267-287.

Vorperian, H. K., Kent, R. D., Lindstrom, M. J., Kalina, C. M., Gentry, L. R., \& Yandell, B. S. (2005). Development of vocal tract length during early childhood: a magnetic resonance imaging study. Journal of the Acoustical Society of America, 117, 338-350.

Wolff, S. E., \& Puts, D. A. (2010). Vocal masculinity is a robust dominance signal in men. Behavioral Ecology and Sociobiology. doi:10.1007/s00265-010-0981-5.

Xue, S. A., \& Hao, G. J. (2003). Changes in the human vocal tract due to aging and the acoustic correlates of speech production: a pilot study. Journal of Speech, Language, and Hearing Research, 46, 689701.

Zahavi, A. (1982). The patterns of vocal signals and the information they convey. Behaviour, 80, 1-8.

Zuckerman, M., \& Miyake, K. (1993). The attractive voice: what makes it so? Journal of Nonverbal Behavior, 17, 119-135.

Carolyn Hodges Simeon received a master's degree in psychology from the College of William and Mary. She is currently pursuing a doctorate in Integrative Anthropological Sciences at the University of California, Santa Barbara. She is interested in sex differences, and her dissertation research will focus on factors affecting the development of sexually dimorphic voice characteristics during puberty. 
Steven J. C. Gaulin earned his B.A. in anthropology and psychology from U.C. Berkeley and his Ph.D. in biological anthropology from Harvard University. He taught anthropology, animal behavior and psychology for 25 years at the University of Pittsburgh, and is now a professor of anthropology at U.C. Santa Barbara, as well as coeditor-in-chief of Evolution and Human Behavior. Sex differences-in anatomy, physiology, behavior and cognition — are his primary research focus. He has seen one-third of the world's extant bird species in the wild, and he plays acoustic finger-style guitar.

David Puts (lab website: http://www.putslab.psu.edu/index.html) is an assistant professor in the Department of Anthropology at Penn State University and is co-funded by the Penn State Children, Youth, and Family Consortium. His research focuses on the neuroendocrine and evolutionary bases of human sexuality and sex differences. He was the 2004 recipient of the Human Behavior and Evolution Society New Investigator Award, was elected member of the International Academy of Sex Research in 2006, and is on the Editorial Board of the journal Archives of Sexual Behavior. 